\title{
Accrued Cost Savings of a Free Clinic Using Quality-Adjusted Life Years Saved and Return on Investment
}

\author{
Jim Sanders, MD, MPH, Marcus Lacey, BS, and Clare E. Guse, MS
}

Introduction: Savings garnered through the provision of preventive services is a form of profit for health systems. Free clinics have been using this logic to demonstrate their cost-savings. The Community-Based Chronic Disease Management (CCDM) clinic treats hypertension using nurse-led teams, clinical protocols, and community-based settings.

Methods: We calculated CCDM's cost-effectiveness from 2007 to 2013 using 2 metrics: Quality-adjusted life years (QALYs) saved and return on investment (ROI). QALYs were calculated using the Clinical Preventive Burden (CPB) score for hypertension care. ROI was calculated by tallying the savings from prevented heart attacks, strokes, and emergency department visits against the total operating costs.

Results: Using conservative assumptions for cost estimates, hypertension care resulted in a value of QALYs saved of $\$ 711,000$ to $\$ 2,133,000$ and an ROI ratio range of 0.35 to 1.20 . Our study shows that when using conservative assumptions to calculate cost-savings, our free clinic did not save money. Costsavings did occur, but the amount was modest, was less than that of cost-inputs, and was not likely captured by any single health entity.

Conclusion: Although free clinics remain a vital health care access point for many Americans, it has yet to be demonstrated that they generate a net savings. (J Am Board Fam Med 2017;30:505-512.)

Keywords: Ambulatory Care Facilities, Chronic Disease, Clinical Protocols, Cost-Benefit Analysis, Health Financing, Health Insurance, Hypertension, Quality-Adjusted Life Years

Millions of Americans lack health insurance and struggle to find affordable access to the health system. For uninsured people with hypertension (HTN), the recurring need for affordable care can be particularly challenging. ${ }^{1}$ Although the uninsured and the insured have similar rates of screening and diagnosis of HTN, the uninsured have lower rates of HTN treatment. ${ }^{2-4}$ This disparity is important because the health consequences of un-

This article was externally peer reviewed.

Submitted 10 March 2017; revised 25 March 2017; accepted 4 April 2017.

From the Department of Family and Community Medicine, Medical College of Wisconsin, Milwaukee (JS, CEG); and Medical College of Wisconsin, Milwaukee (ML).

Funding: This research was funded in part by the Advancing a Healthier Wisconsin endowment at the Medical College of Wisconsin (grants 2007I-06 and 2010I-07), and the Columbia St. Mary's Foundation.

Conflict of interest: none declared.

Corresponding author: Jim Sanders, 1121 E. North Ave, Milwaukee, WI 53211 (E-mail: jsanders@mcw.edu). treated hypertensive disease remain among the leading causes of death in the United States. ${ }^{5-7}$

In 2007, we began the Community-Based Chronic Disease Management Clinic (CCDM) to provide HTN treatment to uninsured patients in Milwaukee. CCDM was designed to eliminate common barriers to health care access for those who are chronically ill and uninsured. Studies have shown that patient outcomes improve when such barriers are addressed. ${ }^{8-12}$ CCDM served neighborhoods in Milwaukee's central city, which is one of the poorest urban areas in the United States. ${ }^{13}$

The CCDM model used community-based, patient-centered resources: neighborhood clinic sites, no appointments, and point-of-care information technology. CCDM purposely co-located its clinics in settings where the patients would be seeking other services, such as food pantries. CCDM intentionally sought out community-based partners to help build patient trust, provide patient-centered 
resources, inform about health care team leadership, provide culturally appropriate health education, and more.

The CCDM clinic was specifically designed not to be a primary care clinic. Instead, CCDM focused exclusively on 3 chronic diseases: high blood pressure (essential type), uncomplicated type 2 diabetes mellitus, and hypercholesterolemia. Patients who had other health complaints were referred to other local clinics for care.

In addition, CCDM put nurses at the head of the health team; this helped keep the human resource costs down. CCDM intentionally used parish nurses who were familiar with the neighborhood in order to better establish trust and rapport with the patient population. Physicians and advanced practice nurses served only as consultants.

The CCDM nurse-led teams followed evidencebased protocols to make clinical decisions. These clinical protocols have been shown to be successful in achieving blood pressure target goals quickly in other settings. All services, including laboratory blood draws and medication dispensing, were provided on site, at no cost to the patients. The background, working, and quality outcomes of CCDM are described in further detail elsewhere. ${ }^{14-16}$

HTN screening and treatment have long been considered effective medical interventions because of the low costs associated with detecting and treating HTN and the relatively high costs of its associated morbidities, such as myocardial infarction and stroke. Three articles describing cost-savings associated with free clinics' care intrigued us, and we wondered whether CCDM's model could also be shown to save costs. ${ }^{17-19}$ Accordingly, we looked at CCDM's savings through two frequently used measurements of health care effectiveness: qualityadjusted life years (QALYs) and return on investment (ROI). Both metrics are commonly used to help shape health programs and allocate resources.

\section{Methods}

\section{Patient Population}

To calculate the number of patients who benefited from CCDM's services, we used a longitudinal sample of patients with HTN over seven years, 2007 through 2013.

\section{CCDM Screened All Its Patients for HTN}

We defined HTN as (1) previous diagnosis of hypertension or (2) systolic blood pressure $>140$
$\mathrm{mmHg}$ or diastolic blood pressure $>90 \mathrm{mmHg}$ for patients without diabetes mellitus, or systolic blood pressure $>130 \mathrm{mmHg}$ or diastolic blood pressure $>80 \mathrm{mmHg}$ for patients with diabetes mellitus, measured on two occasions. We included those patients who had been screened and treated for high blood pressure within the CCDM model of care for at least 1 year ( \pm 10 days). ${ }^{15,16}$ We used the $\mathrm{McNemar}$ test for paired data and logistic regression analyses to assess changes in blood pressure attained at 12 months compared with the initial visit.

\section{Calculating the QALYs Saved}

We first looked at the QALYs saved by HTN treatment through CCDM. Screening and treating HTN are considered to be highly valuable when compared with other interventions for cardiovascular health. We chose a methodology to calculate the QALYs saved that is intuitive and inclusive of the costs, both realized and prevented, generated by a clinic such as CCDM. This method uses a calculated integer called the clinical preventive benefit (CPB). A $\mathrm{CPB}$ score of 1 is considered the least valuable, and a score of 5 is considered the most valuable, for an intervention's costs over the lifetime of a patient population. Treating HTN has achieved a CPB of 5 , thus making it a highly valued intervention. It has been estimated that interventions with a $\mathrm{CPB}$ of 5 save at least 0.09 to 0.65 QALYs per patient. ${ }^{20-23}$ Most studies of this type, including our own, use the lower end of this estimated range when calculating the QALYs saved in their patient population. This seems to be the prudent choice, because no studies can follow patients for the full duration of their natural lives.

Although the successful treatment of HTN is considered a high-value intervention, the resulting value of QALYs saved is a matter of some debate. Monetary values given for a QALY in the United States range from $\$ 50,000$ to $\$ 150,000 .{ }^{24}$ For our calculations, we used a range of commonly quoted QALY values: \$50,000; \$100,000; and \$150,000.

\section{Calculating the ROI}

ROI calculations tally the cost-savings achieved by HTN treatment against the expenditures of treatment. An ROI ratio $>1.0$ is considered cost-beneficial and means the investment yields a net-positive gain. For CCDM, we measured savings that were achieved via three avoided events: myocardial 
infarctions, strokes, and emergency department (ED) visits.

To calculate the savings from avoided myocardial infarctions and avoided strokes, we followed a 4-step algorithm. First, we converted the average blood pressure reductions in the successfully treated CCDM patients into hazard ratios by age for myocardial infarctions and strokes. ${ }^{25}$ Second, we converted the hazard ratios into risk reductions for this same group of patients. Third, we converted the risk reductions that these CCDM patients realized from achieving their blood pressure goals into the predicted number of avoided myocardial infarctions and avoided strokes. ${ }^{26}$ Fourth, we used published costs for treating myocardial infarctions and strokes to calculate the savings associated with these avoided morbidities. ${ }^{27}$

CCDM also achieved savings by providing lowcost access to care in lieu of an ED visit for HTNrelated complaints. A review of the literature describing the experiences of other free clinics in diverting ED visits suggested that $23 \%$ to $49 \%$ of our patients might have sought care at an ED had there not been a CCDM clinic. ${ }^{18,19,28}$ Anecdotal evidence from the CCDM nursing staff suggested a rate of $50 \%$. We used $37.5 \%$ as our figure. A conservative estimate, the figure adjusts for the likelihood that the majority of CCDM patients $(75 \%)$ would have been triaged away from the high-acuity, high-cost ED in favor of a lower-acuity, lower-cost setting, such as an urgent care clinic (UCC).

We calculated the average costs for a HTNrelated ED/UCC visit in the Milwaukee area using data from the Wisconsin Hospital Association's website. ${ }^{29}$ To find the savings accrued by CCDM, we multiplied the number of patients who might have sought care at an ED/UCC by the average ED/UCC costs. These savings were discounted to reflect the likely reimbursement rate of $40 \%$ from Wisconsin's Medicaid program, which most closely represents the income the hospital would have lost by seeing an uninsured CCDM patient.

The expenditures associated with CCDM's services were rather modest because much of the model was built with sustainability in mind. CCDM's expenditures went mainly to pay for the salaries and benefits of the clinic's paid staff. The second-highest cost was for the free distribution of medications. Other costs were rent, utilities, diagnostic laboratory charges, and supplies, as listed in
Table 1. Community-Based Chronic Disease Management Clinic Operational Expense Budget, Fiscal Year 2014

Expense

Amount (\$)

Salaries Nurse practitioner and community health
worker 122,400

Registered nurse (0.4 FTE at \$31/hour)

25,792

Employee benefits (Columbia St. Mary's in-kind)

38,530

Rent (church in-kind)

6,000

Utilities (in-kind estimate)

1,800

Supplies (estimate)

3,600

Pharmacy

68,400

Diagnostics

26,400

Total

$\$ 292,922$

FTE, full-time equivalent.

Table 1. The cost to operate CCDM for 1 year was $\$ 292,922$.

Finally, we calculated the ROI by adding all the savings from avoided myocardial infarctions, strokes, and ED/UCC visits, then dividing that sum by the expenditures associated with CCDM's services.

The Medical College of Wisconsin's institutional review board approved this research under protocol number 00006704 and waived informed consent.

\section{Results}

\section{Patients Who Benefited from CCDM's Services}

From October 24, 2007, through July 31, 2013, CCDM cared for 1,474 patients at 11,809 discrete visits. Of these patients, 1,182 were diagnosed with HTN, and 158 were able to stay with CCDM through 12 months ( \pm 10 days). Approximately $23 \%$ attained their blood pressure goal at the initial visit; $49 \%(n=77)$ were at goal at the 12 -month visit. This improvement is statistically significant $(P<.00005)$.

Regression analysis of these 77 patients showed an average reduction of $18 \mathrm{mmHg}$ for systolic blood pressure from the first visit to the 12-month visit. A similar analysis showed an average reduction of $11 \mathrm{mmHg}$ for diastolic blood pressure between the first visit and the 12-month visit.

More details about the CCDM patients' clinical profiles are shown in Table 2. 
Table 2. Community-Based Chronic Disease

Management Clinic Patient Data from October 24, 2007, through July 31, 2013

1,474 patients had 11,809 visits

158 patients achieved $\geq 1$ year of follow-up

$43 \%$ of the patients had $\geq 5$ visits

$56 \%$ were male

$74 \%$ were categorized as overweight or obese based on BMI measurement

Average age was 48 years

$80 \%$ had HTN diagnosed at the first visit

Average blood pressure at presentation was 151/94 $\mathrm{mmHg}$

BMI, body mass index; HTN, hypertension.

\section{Calculating the QALYs Saved}

Successful long-term treatment of HTN saves an average of 0.09 QALY/person. Of the 1474 CCDM patients screened for HTN, 158 were treated and able to achieve follow-up for at least 1 year. Multiplying the 158 patients by 0.09 equals 14.22 QALYs saved. These results are described in Table 3.

\section{Calculating ROI}

Published studies have shown that patients with decreases in blood pressure similar to those in the 77 patients from CCDM will have corresponding hazard ratios of 0.49 (95\% confidence interval, $0.45-0.53)$ and 0.36 (95\% confidence interval $0.32-0.40)$ for myocardial infarctions and strokes, respectively.

We multiplied the corresponding risk reductions for myocardial infarctions and strokes by their expected incidences by patient age. This calculation shows that 0.45 myocardial infarction and 0.16 stroke had likely been avoided because of HTN treatment by CCDM.

The average costs of treating myocardial infarctions and strokes are known to be $\$ 80,096$ and
$\$ 21,885$, respectively. ${ }^{24} \mathrm{We}$ multiplied these costs by the number of avoided events to arrive at savings of $\$ 36,043$ and $\$ 3,501$, respectively.

Next, we estimated that $37.5 \%$ of CCDM's 11,809 patient visits would have resulted in ED or UCC visits if not for CCDM. Low-, mid-, and high-range charges for HTN-related complaints in Milwaukee-area EDs and UCCs are publicly available, allowing us to calculate savings of $\$ 615,492$ to $\$ 2,196,288$ in health care expenditures avoided because of CCDM. ${ }^{29}$

Finally, we calculated ROI by dividing the sum of CCDM's savings by CCDM's expenditures. This resulted in an ROI ratio of 0.35 using low-end cost estimates, 0.62 using midrange cost estimates, and 1.20 using high-end cost estimates. All ROI calculation data are shown in Table 4.

\section{Discussion}

This study highlights 2 results. First, the CCDM model did not achieve savings greater than its costs using the QALY or the ROI calculations for treatment of HTN. In our calculation for QALYs saved, we found that the monetary value of QALYs saved was less than expenditures over the 7-year period, except when we used the high-end value of $\$ 150,000$ per QALY. Similarly, when we calculated the ROI, we found that all but the high-range estimates resulted in a ratio less than 1.0, meaning that costs exceeded savings.

Second, we had to make numerous assumptions about patient behavior and patient care in order to calculate QALYs saved and the ROI ratio. These assumptions all had a direct influence on the results of the calculations. For example, assumptions were made about fidelity to lifelong blood pressure control when, as is the case with many free clinics, episodic care proved to be the rule rather than the exception; the costs associated with caring for a

Table 3. Quality-Adjusted Life Years Saved with Blood Pressure Intervention

\begin{tabular}{lc}
\hline QALY per patient & 0.09 \\
Patients screened and treated for HTN for at least 1 year (n) & 158 \\
QALYs saved at CCDM & $158 \times 0.09=14.22$ \\
Value of QALYs saved & \\
Low & $14.22 \times \$ 50,000 /$ QALY $=\$ 711,000$ \\
Mid & $14.22 \times \$ 100,000 /$ QALY $=\$ 1,422,000$ \\
High & $14.22 \times \$ 150,000 /$ QALY $=\$ 2,133,000$ \\
\hline
\end{tabular}

CCDM, Community-Based Chronic Disease Management Clinic; HTN, hypertension; QALY, quality-adjusted life year. 


\begin{tabular}{|c|c|c|}
\hline & Calculations & Results \\
\hline \multicolumn{3}{|l|}{ Savings from Blood Pressure Reduction } \\
\hline \multicolumn{3}{|l|}{ MIs } \\
\hline Incidence per 1000 person-years & & 11.4 \\
\hline Hazard ratio for CCDM patients & & 0.48 \\
\hline Risk reduction for $\mathrm{MI}$ & $1-0.48$ & 0.52 \\
\hline MIs avoided per 1000 person-years & $11.4 \times 0.52$ & 5.9 \\
\hline MIs avoided per CCDM's 77 person-years & $0.077 \times 5.9$ & 0.45 \\
\hline Total cost per MI & & $\$ 80,096$ \\
\hline Total MI-related costs avoided by CCDM & $\$ 80,096 \times 0.45$ & $\$ 36,043$ \\
\hline \multicolumn{3}{|l|}{ Strokes } \\
\hline Incidence per 1000 person-years & & 3.3 \\
\hline Hazard ratio for CCDM patients & & 0.36 \\
\hline Risk reduction for strokes & $1-0.36$ & 0.64 \\
\hline Strokes avoided per 1000 person-years & $3.3 \times 0.64$ & 2.1 \\
\hline Strokes avoided per CCDM's 77 person-years & $0.077 \times 2.1$ & 0.16 \\
\hline Total cost per stroke & & $\$ 21,885$ \\
\hline Total stroke-related costs avoided by CCDM & $\$ 21,885 \times 0.16$ & $\$ 3,501$ \\
\hline Subtotal & $\$ 36,043+\$ 3,501$ & $\$ 39,544$ \\
\hline \multicolumn{3}{|l|}{ Savings from emergency department visits avoided } \\
\hline Total patient visits to $\mathrm{CCDM}$ & & 11,809 \\
\hline Patients likely to use ED in lieu of CCDM (\%) & & 37.5 \\
\hline \multicolumn{3}{|l|}{$\begin{array}{l}\text { Average ED/UCC visit charges in Milwaukee } \\
\text { metro area* }\end{array}$} \\
\hline Low & & $\$ 348$ \\
\hline Mid & & $\$ 623$ \\
\hline High & & $\$ 1,239$ \\
\hline \$348 Average ED/UCC visit costs in Milwaukee & Charge $\times 40 \%$ & \\
\hline Low & & $\$ 139$ \\
\hline Mid & & $\$ 249$ \\
\hline High & & $\$ 496$ \\
\hline CCDM ED/UCC visits avoided & $11,809 \times 0.375$ & 4,428 \\
\hline \multicolumn{3}{|l|}{ Subtotal } \\
\hline Low & $4,428 \times \$ 139$ & $\$ 615,492$ \\
\hline Mid & $4,428 \times \$ 249$ & $\$ 1,102,572$ \\
\hline High & $4,428 \times \$ 496$ & $\$ 2,196,288$ \\
\hline \multicolumn{3}{|l|}{ Total savings } \\
\hline Low & $\$ 39,544+\$ 615,492$ & $\$ 655,036$ \\
\hline Mid & $\$ 39,544+\$ 1,102,572$ & $\$ 1,142,116$ \\
\hline High & $\$ 39,544+\$ 2,196,288$ & $\$ 2,235,832$ \\
\hline Total CCDM expenditures & $\$ 292,922 /$ year $\times 7$ years & $\$ 1,840,454$ \\
\hline Return on investment & $\$ 1,142,116 / \$ 1,840,454$ & $0.62(0.35-1.20)$ \\
\hline
\end{tabular}

*Emergency department (ED) and urgent care clinic (UCC) charges were used to calculate the composite charge estimate, weighted $25 \%$ and $75 \%$, respectively.

CCDM, Community-Based Chronic Disease Management Clinic; MI, myocardial infarction.

patient who had a myocardial infarction or stroke; the proportion of patients who would seek care from an ED/UCC in lieu of CCDM; the proportion of those patients who would be seen chiefly for HTN; the proportion of those patients who would be routed to the ED (high cost) or UCC (lower cost); and that patients seeking care at the ED/ UCC would be evenly distributed throughout the Milwaukee health system. In addition, our calculations accounted for only 2 HTN-related morbidities (heart attacks and strokes), but other wellknown HTN morbidities could also have been 
included, such as kidney failure, dementia, congestive heart failure, and cardiac arrhythmias.

We carefully assessed the validity of each of our assumptions. When there was ample uncertainty, we tried to include a range of costs, such as those at the low, middle, and high ranges, to highlight the various possible outcomes. When the assumption was more of a judgment call, such as deciding what time period to use when defining successful HTN control in our patients, we erred on the side of a conservative estimate. Other studies performing similar cost-savings analyses used less conservative assumptions and broader inclusion criteria, such as counting a one-time clinical encounter for blood pressure screening as an intervention of significant value. Not surprisingly, by using less rigorous inclusion criteria, these studies demonstrated high QALYs saved and high ROI ratios for overall net cost-savings. ${ }^{17-19}$

Despite our study's results, continued operation of CCDM is warranted. The goal of the clinic is to provide free, high-quality management of chronic diseases to the uninsured population of Milwaukee. This study, along with other previous publications about CCDM's work, have demonstrated that it has fulfilled its original purpose. ${ }^{15,16}$

Second, this study evaluates whether management of HTN alone achieved cost-savings at CCDM. It is important to keep in mind that several services at CCDM are associated with a high CPB rating (eg, screening and managing uncomplicated type 2 diabetes mellitus and hypercholesterolemia) and thus might represent significant QALYs saved. Ideally, these services could be analyzed using similar methodology to provide a more accurate representation of total cost-savings at CCDM. However, setting the inclusion criteria for the care of uncomplicated type 2 diabetes mellitus and hypercholesterolemia at one year, as we did with HTN care, resulted in too few patients being significantly affected by these interventions to account for any meaningful savings by CCDM. Adjusting the study's criteria to favor the inclusion of more patients would weaken our intent to rigorously test the QALY and ROI economic model.

In a similar manner, patients may have significantly benefited from CCDM's services even if they were lost to follow-up before the one-year treatment date. As a result, many areas of cost savings within CCDM's treatment services were likely not captured by our study's rigorous design.
Last, from a patient perspective, unmeasured economic savings may have accrued from CCDM's presence. Patients incur many costs associated with seeking and receiving health care services beyond the direct out-of-pocket expenses. For example, indirect costs might include decreased productivity, missed work, transportation fees, and child care. These types of costs can be especially burdensome for poorer patient populations such as those found in CCDM's catchment neighborhoods. The ED likely represents a poor cost-to-benefit ratio for these patients because of long wait times, distance to travel and transportation issues, resultant lost productivity, and the direct costs of prescription medications. CCDM avoids these cost by having shorter wait times, community partnerships to improve proximal accessibility, and free medications dispensed on site. Thus, the benefits to CCDM's patients result in not only direct monetary savings but also indirect savings of enhanced productivity, improved quality of life, and the value of better follow-up care through CCDM's model. These are very real savings but fall outside of those savings that would likely accrue to any single health system and, as such, are outside the scope of this study.

It remains uncertain how legitimate it is to count any savings generated from a free communitybased clinic when health care markets are highly competitive and often overlap. When we speak of savings, we imply that these savings would flow to just one entity. The geographic overlap of the health systems around CCDM's catchment area, however, means that CCDM patients were near a number of EDs and UCCs from numerous health systems. Thus, the savings generated from CCDM's work could easily have been realized by different health systems in addition to the one that directly supported CCDM. This is likely to be the case in other urban areas around the country. Until health systems align with one another and put themselves in a better position to capture the savings from the support of community-based endeavors such as free clinics, charity care will likely remain just that.

\section{Limitations}

We used the National Heart, Lung, and Blood Institute's heart attack and stroke incidence rates from the 2006 chart book, which might not accurately reflect the rates during our study period. Furthermore, we did not collect racial data, so we 
are unable to account for race in our estimates of myocardial infarction and stroke incidence.

\section{Implications for Policy and Practice}

Cost-savings can occur through the provision of free care, but they are likely modest and are not likely captured by any single health entity. Thus, although free clinics remain a vital access point to health care for many Americans, using QALYs and ROI for modeling in economic analyses does not consistently demonstrate that they generate a net savings when rigorous inclusion criteria are used, and they should be used with caution for this purpose.

\section{Conclusions}

This study highlights the savings associated with CCDM's work in two separate but related ways: QALYs saved and ROI. Using conservative assumptions to calculate savings, our study showed that CCDM did not save costs when measured against its expenditures. The savings from QALYs or HTN-related morbidities avoided would likely have been greater had the number of successfully treated patients with HTN been higher and had other chronic disease services, such as screening and managing uncomplicated type 2 diabetes mellitus and hypercholesterolemia, been considered. Thus, although CCDM remains a novel way of delivering chronic disease care, it has yet to demonstrate cost-savings with rigorous inclusion criteria and by using QALYs or ROI as economic models.

The CCDM clinical staff includes Brenda Buchanan, RN, Julia Means, RN, Nancy Leahy, APNP, Christy Tolbert, Bill Solberg, MSW, David Goines, Johnny Ayers, Robert Ramerez, and Carla Harris, RN. The authors thank Jeffrey Whittle, MD, $\mathrm{MPH}$, and Michael Gauger for their review of the manuscript.

To see this article online, please go to: http://jabfm.org/content/ 30/4/505.full.

\section{References}

1. Nardin R, Sayah A, Lokko H, Woolhandler S, McCormick D. Reasons why patients remain uninsured after Massachusetts' health care reform: a survey of patients at a safety-net hospital. J Gen Intern Med 2012;27:250-6.

2. Wilper AP, Woolhandler S, Lasser KE, McCormick D, Bor DH, Himmelstein DU. Hypertension, diabetes, and elevated cholesterol among insured and uninsured U.S. adults. Health Aff (Millwood) 2009; 28:1151-9.

3. Duru OK, Vargas RB, Kermah D, Pan D, Norris $\mathrm{KC}$. Health insurance status and hypertension monitoring and control in the United States. Am J Hypertens 2007;20:348-53.

4. Brooks EL, Preis SR, Hwang SJ, et al. Health insurance and cardiovascular disease risk factors. Am J Med 2010;123:741-7.

5. Centers for Disease Control and Prevention. CDC WONDER: Underlying cause of death, 1999-2015 [databaseon theInternet].Availablefrom:http://wonder. cdc.gov/controller/datarequest/D76;jsessionid = B9D7603BCE2EA9F7F9B8275AA1D23C9E. Accessed February 9, 2016.

6. Murphy SL, Xu J, Kochanek KD. Deaths: final data for 2010. Natl Vital Stat Rep 2013;61:1-117.

7. Ford ES, Capewell S. Proportion of the decline in cardiovascular disease mortality due to prevention versus treatment: public health versus clinical care. Annu Rev Public Health 2011;32:5-22.

8. Shelley D, Tseng TY, Andrews H, et al. Predictors of blood pressure control among hypertensives in community health centers. Am J Hypertens 2011;24: $1318-23$.

9. Blanchard J, Ogle K, Thomas O, Lung D, Asplin B, Lurie N. Access to appointments based on insurance status in Washington, D.C. J Health Care Poor Underserved 2008;19:687-96.

10. Hadley J, Cunningham P. Availability of safety net providers and access to care of uninsured persons. Health Serv Res 2004;39:1527-46.

11. Persell SD, Osborn CY, Richard R, Skripkauskas S, Wolf MS. Limited health literacy is a barrier to medication reconciliation in ambulatory care. J Gen Intern Med 2007;22:1523-6.

12. Russell BE, Gurrola E, Ndumele C, et al. Perspectives of non-Hispanic black and Latino patients in Boston's urban community health centers on their experiences with diabetes and hypertension. J Gen Intern Med 2010;25:504-9.

13. Tolan T, Herzog K. Poverty numbers spike in Milwaukee. Milwaukee Journal Sentinel, 2011 Sept 21. Available from: http://www.jsonline.com/news/ milwaukee/Poverty-numbers-spike-in-Milwaukee. html. Accessed March 24, 2016.

14. Sanders J, Solberg B, Gauger M. Breaking barriers to care: a community of solution for chronic disease management. J Am Board Fam Med 2013;26:311-5.

15. Sanders J, Guse C, Onuoha B. A new model for managing hypertension in an uninsured population. J Community Health 2013;4:44-9.

16. Sanders J, Guse CE. Reaching urban poor hypertensive patients: a novel model of chronic disease care versus a traditional fee-for-service approach. J Prim Care Community Health 2017;8:14-9. 
17. Oriol NE, Cote PJ, Vavasis AP, et al. Calculating the return on investment of mobile healthcare. BMC Med 2009;7:27.

18. Bicki A, Silva A, Joseph V, et al. A nurse run walk-in clinic: cost effective alternative to non-urgent emergency department use by the uninsured. J Community Health 2013;38:1042-9.

19. Song Z, Hill C, Bennet J, et al. Mobile clinic in Massachusetts associated with cost savings from lowering blood pressure and emergency department use. Health Aff (Millwood) 2013;32:36-44.

20. Maciosek MV, Edwards NM, Nelson WW, Davis MK, McGree DA. Hypertension screening: technical report prepared for the National Commission On Prevention Priorities. Version 06.1; last updated August 19, 2008. Available from: www.prevent.org/ data/files/initiatives/hypertension_screening_and_ treatment.pdf. Accessed March 19, 2017.

21. Maciosek MV, Coffield AB, McGinnis JM, et al. Methods for priority setting among clinical preventive services. Am J Prev Med 2001;21:10-19.

22. Maciosek MV, Coffield AB, Edwards NM, Flottemesch TJ, Goodman MJ, Solberg LI. Priorities among effective clinical preventive services. Am J Prev Med 2006;31:52-61.

23. Coffield A, Maciosek M, McGinnis JM, et al. Priorities among recommended clinical preventive services. Am J Prev Med 2001;21:1-9.
24. Neumann P, Cohen J, Weinstein M. Updating cost effectiveness-the curious resilience of the $\$ 50,000$ per-QALY threshold. N Engl J Med 2014;371: 796-7.

25. Lewington S, Clarke R, Qizilbash N, Peto R, Collins R; Prospective Studies Collaboration. Age-specific relevance of usual blood pressure to vascular mortality: a meta-analysis of individual data for one million adults in 61 prospective studies. Lancet 2002;360: 1903-13.

26. National Heart, Lung, and Blood Institute. Incidence and prevalence, 2006 chart book on cardiovascular and lung diseases. Bethesda, MD: National Institutes of Health; 2006. Available from: www. nhlbi.nih.gov/sites/www.nhlbi.nih.gov/files/06_ip_ chtbk.pdf. Accessed April 3, 2016.

27. O'Sullivan AK, Rubin J, Nyambose J, Kuznik A, Cohen DJ, Thompson D. Cost estimation of cardiovascular disease events in the us. Pharmacoeconomics 2011;29:693-704.

28. Gertz AM, Frank S, Blixen CE. A survey of patients and providers at free clinic across the US. J Community Health 2011;36:83-93.

29. Price Point, Wisconsin Hospitals Accountable for Transparency, Wisconsin Hospital Association. Emergency/urgent care services. Available from: www. wipricepoint.org/Report_EDS.aspx. Accessed April 8, 2016. 